\title{
Implicaçóes das cirurgias de câncer de mama nas atividades profissionais
}

\author{
Mirella Dias ${ }^{a}$, Kamilla Zomkowski ${ }^{\text {b }}$ Fernanda Alessandra Silva Michels ${ }^{c}$, \\ Fabiana Flores Sperandio ${ }^{\mathrm{a}}$
}

${ }^{a}$ Universidade Federal de Santa Catarina - UFSC, Florianópolis, SC, Brasil.

${ }^{b}$ Universidade Estadual de Santa Catarina - UDESC, Florianópolis, SC, Brasil.

'Universidade de São Paulo - USP, São Paulo, SP, Brasil.

\begin{abstract}
Resumo: Introdução: O câncer de mama representa $25 \%$ de todos os tipos de câncer, sendo o de maior incidência na população feminina. Com o avanço dos tratamentos e diagnósticos precoces, as taxas de sobrevida têm aumentado e tal contexto reflete nos afastamentos laborais, tendo em vista que a maioria das mulheres se encontra em idade produtiva. Os principais motivos responsáveis pelos afastamentos do trabalho são as complicações físicas decorrentes do tratamento cirúrgico. Objetivo: Objetivou-se investigar as implicações cirúrgicas do tratamento do câncer de mama na atividade profissional, caracterizar o número de afastamentos laborais e investigar a relação entre o tipo de cirurgia nos afastamentos do trabalho e seus principais motivos. Método: Estudo transversal envolvendo 74 mulheres diagnosticadas com câncer de mama. Para a coleta dos dados, foi utilizada uma entrevista semiestruturada, que abordou as questões clínico-cirúrgicas, os dados sociodemográficos, o comportamento laboral e o tratamento fisioterapêutico. Os dados foram organizados no Microsoft Excel mediante frequências e o teste de qui-quadrado, sendo considerado $\mathrm{p} \leq 0,05$. Resultados: $\mathrm{O}$ lado esquerdo foi o mais acometido pela doença (51\%), a cirurgia radical modificada do tipo Madden foi a mais comum (50\%) e 93,2\% submeteram-se à linfadenectomia axilar. As complicações operatórias mais frequentes foram dor, problemas cicatriciais, alteração da sensibilidade, limitação da ADM, linfedema e seroma. Apenas 58\% delas fizeram fisioterapia e 60\% afastaram-se das atividades profissionais, 23\% abandonaram o trabalho, 26\% modificaram as funções do trabalho e 14\% se aposentaram por motivo de doença. Conclusão: Este estudo sugere a existência de uma relação direta do tratamento sobre os afastamentos laborais.
\end{abstract}

Palavras-chave: Mastectomia, Emprego, Neoplasias da Mama, Retorno ao Trabalho.

\section{Breast cancer surgery effect over professional activities}

\begin{abstract}
Introduction: Breast cancer is responsible for $25 \%$ of all cancers and is the most prevalent in the female population. Due to treatment advances and early diagnoses, survival rates have improved, however this condition impacts work absenteeism due to the productive age of these women. The main factors responsible for work absenteeism are physical complications due to surgical treatment. Objective: The aim of this study is to investigate the effects of surgical breast cancer treatments on occupation, to characterize the degree of work absenteeism and to investigate the type of relation between surgical technique and absenteeism's main causes. Method: Cross-sectional study with 74 women diagnosed with breast cancer. A semi-structured interview was used to collect information regarding surgical and clinical aspects, sociodemographic data, work behavior and physical therapy treatments. The data was organized on Microsoft Excel and analyzed by frequency and chi-squared test. The significance level considered was $\mathrm{p} \leq 0.05$. Results: Breast cancer was most common on the left side ( $51 \%)$, Madden modified radical mastectomy was the most common (50\%) and lymph node resection was present in $93.2 \%$ of cases. The most frequent
\end{abstract}

Autor para correspondência: Kamilla Zomkowski, Laboratório de Saúde da Mulher, Centro de Ciências da Saúde e do Esporte, Universidade do Estado de Santa Catarina, Rua Pascoal Simone, 358, Coqueiros, CEP 88080-350, Florianópolis, SC, Brasil, e-mail: kamillazomkowski@gmail.com Recebido em Nov. 30, 2015; $1^{\text {a }}$ Revisão em Abr. 6, 2016; 2ª Revisão em Jul. 7, 2016; Aceito em Ago. 4, 2016. 
post-surgery complications were pain, problems with scarring, sensitivity alterations, ROM limitation, lymphedema and seroma. Only $58 \%$ of women were treated with physical therapy and $60 \%$ withdrew from professional activities, $23 \%$ abandoned work, $26 \%$ changed their work role and $14 \%$ retired due to the disease. Conclusion: The present study suggests the existence of a direct relation between treatment and work absenteeism.

Keywords: Mastectomy, Employment, Breast Neoplasms, Return to Work.

\section{Introdução}

O câncer de mama representa $25 \%$ de todos os tipos de câncer, tornando-o um dos maiores problemas de saúde pública, na população feminina (INTERNATIONAL..., 2012). De acordo com o Instituto Nacional de Câncer (2016), confirmaram-se mais de 55 mil casos de neoplasias de mama, estando estes mais concentrados nas Regióes Sudeste e Sul, com 71,18/100 mil e 70,98/100 mil, respectivamente. Somente no Estado de Santa Catarina, entre os meses de janeiro e junho de 2015, foram identificados 1.253 mulheres com a doença (INSTITUTO..., 2016).

Com o avanço das técnicas de tratamento e dos diagnósticos cada vez mais precoces, a taxa de sobrevida tem aumentado, nos países desenvolvidos, atingindo valores de até $85 \%$, nos primeiros cinco anos após o diagnóstico. Nos países em desenvolvimento, como o Brasil, tais valores oscilam entre 50 e $60 \%$ (INSTITUTO..., 2014). O aumento desta sobrevida poderá refletir nos afastamentos laborais, tendo em vista que, na maioria das vezes, essas mulheres estão em plena idade produtiva.

A literatura identifica como complicaçôes recorrentes, tanto da abordagem cirúrgica radical quanto da conservadora, o linfedema, a dor na incisão cirúrgica e a diminuição de força/amplitude de movimento (ADM) dos membros superiores (MMSS). Tais complicaçóes prejudicam, em curto e em longo prazo, as atividades de vida diária (AVD's), o desempenho profissional e a habilidade para realizar papéis e tarefas distintas (RHIE; JEONG; WON, 2013).

A cronicidade destas complicaçôes poderá comprometer a atividade profissional dada a demora em retornar ao trabalho. As evidências demonstram que as mulheres levam cerca de 340 dias, em média, para retornarem as atividades de trabalho, considerando, nestes casos, o tempo de dois anos após o término dos tratamentos (ROELEN et al., 2011). Nesse mesmo período, $13 \%$ permanecem ainda em afastamento pela doença e $11 \%$ se aposentam precocemente, em razão da presença da dor e de fadiga (CARLSEN et al., 2014; LINDBOHM et al., 2014).
Para algumas mulheres, o retorno à atividade profissional, por vezes, é interpretado como uma situação de estresse, em função da necessidade de conciliar a nova condição com a vida profissional. $\mathrm{Na}$ literatura internacional, as barreiras estáo na sobrecarga físico-emocional do tratamento em si, na terapêutica de um câncer em estágio avançado e, ainda, nas dificuldades de relacionamento entre colegas e superiores (ISLAM et al., 2014; FANTONI et al., 2010; SALONEN et al., 2011). Em contrapartida, alguns estudos sugerem que a manutenção da atividade profissional no transcorrer do diagnóstico e do tratamento da doença promove melhor qualidade de vida ao longo do processo terapêutico (SALONEN et al., 2011; TIMPERI et al., 2013; DORLAND et al., 2016).

A literatura atesta a necessidade da realização de estudos que caracterizem a forma como estas mulheres, sobreviventes ao câncer de mama, se comportam profissionalmente, dado que tais informaçóes sáo amplamente exploradas em cenários internacionais (BARNES et al., 2014; BLINDER et al., 2012; COOPER et al., 2012). Por fim, entende-se ser oportuno refletir sobre as condutas nacionais e as açôes preventivas na atual conjuntura das açóes em saúde. Os estudos objetivam reduzir o impacto físico, psíquico e laboral, para que o retorno laboral deste coletivo seja rápido e seguro (ISLAM et al., 2014).

Face ao exposto, o objetivo deste estudo é investigar as implicações das cirurgias no tratamento do câncer de mama na atividade profissional da mulher, caracterizar tal população quanto ao número de afastamentos laborais e, por fim, investigar a relação entre o tipo de cirurgia nos afastamentos do trabalho e as principais queixas por elas identificadas.

\section{Método}

Trata-se de um estudo transversal e descritivo, envolvendo 74 mulheres residentes da grande Florianópolis-SC, diagnosticadas com câncer de mama e que realizaram sua primeira consulta no CEPON (Centro de Pesquisas Oncológicas, Santa Catarina). A pesquisa teve aprovação Comitê de Ética de Pesquisa em Seres Humanos da Universidade Federal de Santa Catarina e do Centro de Estudos 
e Pesquisas Oncológicas, sob a resolução 196/96 e 251/97 do CNS, sendo aprovado na Universidade Federal de Santa Catarina - UFSC, sob o protocolo $n^{\circ}$ 088/2004. O estudo foi conduzido com o consentimento expresso das usuárias, obtido a partir da leitura e da assinatura do Termo de Consentimento Livre e Esclarecido.

A amostra foi obtida por conveniência, durante o turno vespertino, junto ao setor de registro de consultas do CEPON. As coletas foram realizadas pelo mesmo pesquisador em um dos ambulatórios desta instituição e, quando necessário, no próprio domicílio das participantes. Os critérios de inclusão para as participantes deste estudo foram: ter mais de 18 anos; estar realizando tratamento ambulatorial; não apresentar metástases; ter sido submetida a algum tipo de intervenção cirúrgica para a retirada da neoplasia mamária, e exercer alguma atividade profissional, remunerada ou náo, previamente ao tratamento.

Para a coleta, foi utilizada uma entrevista semiestruturada, que envolvia questóes abertas e de múltipla escolha previamente testadas com cinco mulheres, que estavam em consulta com um dos médicos oncologistas do setor. Para a descrição do perfil sócio-demográfico, considerou-se a data de nascimento, a idade, o endereço, o telefone, o estado civil e a escolaridade. As informaçóes sobre os dados clínico-cirúrgicos foram obtidas via entrevista ou em consultas aos prontuários médicos utilizados rotineiramente. Para tanto, optou-se pela análise do tipo de cirurgia realizada (radical ou conservadora), da presença de queixas pós-cirúrgicas (diminuição do movimento, linfedema, diminuição da sensibilidade de dor), da realização de fisioterapia pós-operatória, do comportamento na atividade laboral e seus respectivos motivos, uma análise baseada em estudo prévio de Fantoni et al. (2010).

Os registros das entrevistas foram armazenados em um banco de dados no programa Microsoft Excel $^{\circ}$. Posteriormente, os dados organizados foram analisados com o programa SPSS ${ }^{\oplus}$ statistical package for social sciences versão 19.0. $\mathrm{Na}$ análise inferencial do desfecho 'afastamento da atividade profissional', a verificação do relacionamento desta com outras variáveis qualitativas foi mensurada pela estatística do teste qui-quadrado e foi considerado $\mathrm{p} \leq 0,05$.

\section{Resultados}

Dentre as 137 mulheres diagnosticadas com câncer de mama registradas no CEPON, a amostra final foi composta por $54 \%$ da amostra inicial. O óbito foi registrado em $29 \%$ e $13 \%$ delas náo residiam mais em Florianópolis, sendo que $4 \%$ mulheres não aceitaram participar da pesquisa (Figura 1).

Quanto às características sócio-demográficas, a idade das entrevistadas mostrou grande variabilidade, elas estavam na faixa de 20 a 92 anos $( \pm 56,5)$. A maioria da amostra foi composta por mulheres caucasianas (95\%), casadas (53\%) e com o Ensino Fundamental incompleto (32\%). Em relação à cirurgia para o tratamento do câncer de mama, o

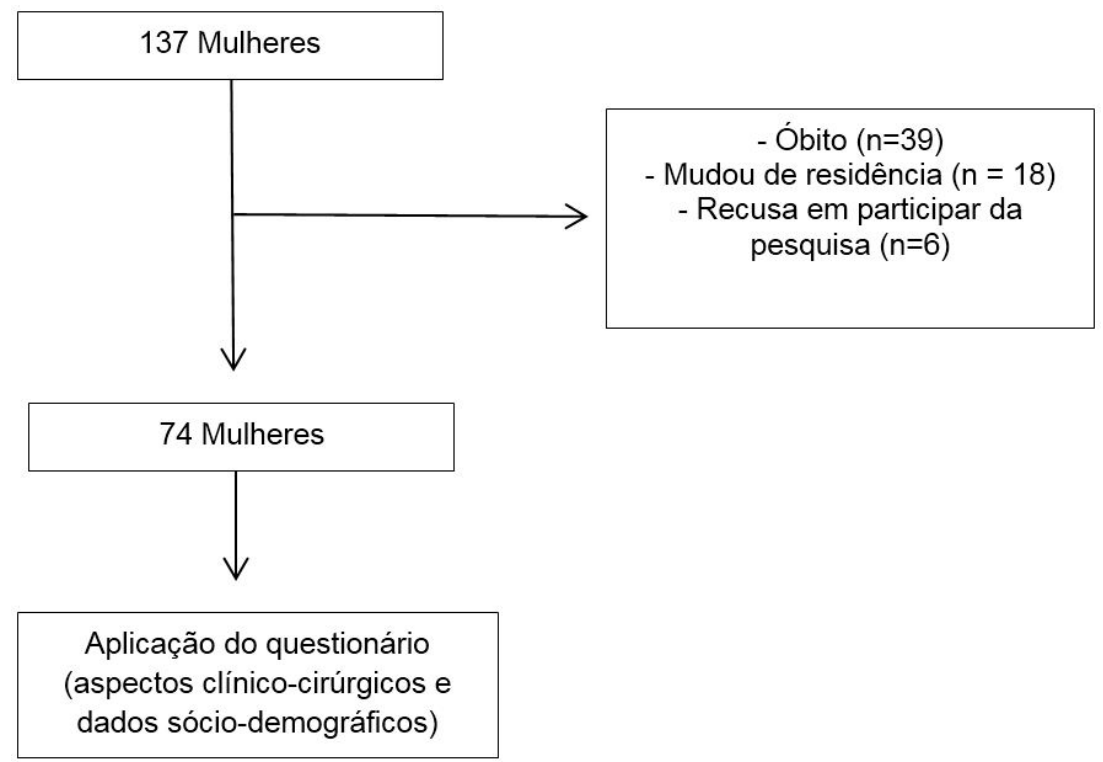

Figura 1. Esquematização da amostra inicial e os motivos da perda amostral. 
lado esquerdo foi o mais acometido (51\%) e metade da amostra foi tratada com a mastectomia radical modificada do tipo Madden, sendo que quase a totalidade das mulheres, no presente estudo, submeteu-se ao procedimento de linfadenectomia axilar (93\%) (Tabela 1).

No que diz respeito às complicaçóes por elas relatadas, as mais frequentes foram: a dor, os problemas cicatriciais, as alteraçôes da sensibilidade, a limitação na $\mathrm{ADM}$, o linfedema e o seroma. Quanto ao número de queixas, em torno de $38 \%$ das mulheres do presente estudo referiram ter nenhuma queixa, seguidas de $33 \%$ que relataram pelo menos uma queixa. Em complemento, cerca de $17 \%$ delas mencionaram três complicaçóes concomitantes, enquanto que $13 \%$ destas mulheres citaram apenas duas queixas.

Tabela 1. Características cirúrgicas das pacientes avaliadas.

\begin{tabular}{lc}
\hline \multicolumn{1}{c}{ Características Cirúrgicas } & Frequências (\%) \\
\hline Lado operado & \\
Esquerdo & 51,38 \\
Direito & 45,83 \\
Bilateral & 2,79 \\
Tipo de Cirurgia & \\
Pattey & 8,33 \\
Madden & 50 \\
Halsted & 2,79 \\
Tumorectomia & 5,55 \\
Quadrantectomia & 33,33 \\
Linfanedectomia Axilar & 93,24 \\
\hline
\end{tabular}

Durante a análise dos questionários, constatou-se que apenas $58 \%$ das mulheres entrevistadas realizaram tratamento fisioterapêutico pós-operatório e $60 \%$ tiveram que se afastar das atividades profissionais após a cirurgia. Observou-se que $26 \%$ das mulheres reduziram suas atividades no trabalho do lar. Entre as que conseguiram retornar às atividades profissionais, cerca de $26 \%$ tiveram que se adaptar em suas funçôes e 23\% abandonaram seus empregos para se dedicar às atividades domésticas. Os trabalhos manuais tiveram a participação por $9 \%$ das mulheres e apenas uma estava acamada (2,3\%). Além dos afastamentos e das modificaçóes na atividade profissional, a aposentadoria, por motivo do câncer de mama, foi verificada em $14 \%$ das mulheres.

Os principais motivos que levaram ao afastamento das mulheres de suas atividades profissionais foram a necessidade de utilizar os membros superiores, mais especificamente a mão, e as queixas emocionais decorrentes da demanda de exames e consultas clínicas. Sobre a quantidade de motivos que fizeram as mulheres abandonarem ou modificarem a atividade profissional, cerca de $56 \%$ delas relataram três ou mais motivos, $30 \%$ delas apontaram um único motivo e dois motivos foram referidos em $14 \%$ dos casos.

Ao analisar a Tabela 2 , observa-se que náo houve diferenças estatisticamente significantes $(\mathrm{p}=0,72 \mathrm{e}$ $\mathrm{p}=0,15)$ na distribuição do afastamento da atividade profissional quando foram relacionados com a técnica cirúrgica e a fisioterapia pós-operatória. Em relação ao linfedema, na distribuição do afastamento da atividade profissional, a significância estatística encontrada foi marginal $(\mathrm{p}=0,052)$.

Tabela 2. Distribuição do afastamento da atividade profissional relacionado a técnica cirúrgica, fisioterapia pós-operatória e existência de linfedema, e resultados dos testes de qui-quadrado.

\begin{tabular}{lccccc}
\hline \multicolumn{2}{c}{ Afastamento (n / \%) } & \multicolumn{2}{c}{$\mathbf{X}^{2}$} & $\mathrm{p}^{*}$ \\
\hline Técnica Cirúrgica & Não & Sim & Total & 0,13 & 0,72 \\
\hline Radicais & 17 & 27 & 44 & & \\
Conservadoras & $38,6 \%$ & $61,4 \%$ & $100 \%$ & & \\
Fisioterapia Pós-operatória & 12 & 16 & 28 & & \\
Não & $42,9 \%$ & $57,1 \%$ & $100 \%$ & & 0,15 \\
& 16 & 15 & & 2,07 & \\
Sim & $51,6 \%$ & $48,4 \%$ & $100 \%$ & & \\
& 15 & 28 & 43 & & \\
Linfedema & $34,9 \%$ & $65,1 \%$ & $100 \%$ & & \\
Não & 27 & 29 & 56 & 3,78 & \\
& $48,2 \%$ & $51,8 \%$ & $100 \%$ & & \\
Sim & 4 & 14 & 18 & & \\
& $41,9 \%$ & $58,1 \%$ & $100 \%$ & & \\
\hline
\end{tabular}

$* \mathrm{p}<0,05$. 


\section{Discussão}

O objetivo deste estudo foi investigar as implicações das cirurgias do câncer de mama na atividade profissional da mulher, na medida em que mais da metade delas se afastou da atividade profissional após o tratamento cirúrgico. A redução das atividades no trabalho doméstico também foi relatada e, ainda, entre as mulheres que conseguiram retornar ao trabalho, uma parte delas teve que adaptar as funçôes laborais à sua nova condição de saúde. $\mathrm{O}$ abandono da atividade profissional por elas mencionado deu lugar à dedicação às tarefas domiciliares e uma parcela delas se aposentou pelo câncer de mama, reforçando o compromisso com a melhora de sua saúde e a complexa segurança exigida.

No presente estudo, náo houve diferenças estatisticamente significantes em relação ao afastamento da atividade profissional e a técnica cirúrgica adotada; no entanto, quando analisadas individualmente, observa-se que as técnicas usadas trouxeram, em maior ou menor grau, limitaçóes físicas às mulheres que se afastaram de seus trabalhos, $o$ que reforça a necessidade de monitoramento desta relação: saúde laboral e oncologia.

Esta pesquisa apresentou uma taxa relativamente alta de afastamentos da atividade profissional, estando estes relacionados com o maior número de queixas físicas. Dentre os motivos por elas relatados sobre a decisão de afastar-se do trabalho, o medo de se machucar, a diminuição de força nos MMSS, a menor agilidade, a indisposição para trabalhar, a sensação de braço pesado, a perda do movimento, a dor e, ainda, a elevada frequência de exames contribuíram para esta conduta. Esses achados são corroborados pelos estudos de Fantoni et al. (2010), Lilliehorn et al. (2013) e Lindbohm et al. (2014).

Das mulheres entrevistadas, a maior parte estava afastada da atividade profissional (60\%), sendo que a cirurgia radical foi observada em $63 \%$ delas. As cirurgias realizadas para o tratamento do câncer de mama têm como objetivo comum o propósito de remover o tecido maligno, cuja principal diferença reside na extensão da remoção de tecidos adjacentes (VERONESI et al., 2002). Atualmente, há evidências científicas suficientes de que as cirurgias conservadoras possam ser realizadas com a mesma segurança das cirurgias radicais (COWHER et al., 2014; CHEN et al., 2014). Não podemos desconsiderar que o uso das cirurgias conservadoras diminui as complicações pós-operatórias por serem menos invasivas e terem reduzidos efeitos negativos na qualidade de vida destas mulheres, já que preservam os tecidos adjacentes e contribuem para que não haja prejuízo funcional de MMSS (PYFER et al., 2015; CHEN et al., 2014).

Ao investigar a relação entre o tipo de cirurgia e o afastamento do trabalho, as principais queixas por elas identificadas foram as complicaçôes físicas, das quais se podem citar o linfedema, a dor, as alterações cicatriciais, o seroma, as restriçóes na ADM da cintura escapular e as alteraçóes da sensibilidade. Tais resultados lançam desafios à equipe interdisciplinar na medida em que a dor presente na área da incisão cirúrgica e os consequentes problemas cicatriciais pós-operatórios são alterações físicas, igualmente comprometedoras, e bem documentadas em estudos anteriores (EDWARDS et al., 2013). Esta dor, se persistente, sugere que tenha ocorrido o desenvolvimento de alterações nos processos moduladores da dor em nível de sistema nervoso central (EDWARDS et al., 2013).

Outra complicação bastante comum foi o linfedema, estando este presente em $78 \%$ das mulheres da amostra. Paralelamente, em 33\% dos casos, o linfedema esteve relacionado com a cirurgia da mama e náo com outras modalidades de tratamento do câncer de mama, como o esvaziamento axilar ou a radioterapia, os quais podem promover tal condição (TSAI et al., 2009). Em relação a isso, a literatura descreve que o linfedema ocorre dentro do primeiro ano e seu aparecimento poderá ocorrer em distintos momentos (TSAI et al., 2009; MONLEON et al., 2015; TOGAWA et al., 2014). Recentemente, Cowher et al. (2014) sugeriram que, a cada oito linfonodos retirados, há uma chance 1,5 vez maior de desenvolver essa condição. Isto posto, sugerem-se mais investigaçôes sobre o processo de aparecimento do linfedema e os possíveis efeitos na dinâmica laboral, em diferentes momentos do processo de reabilitação.

Vale considerar que as complicações físicas dificultam a realização das AVD's. Tal condição está atrelada à técnica cirúrgica empregada, sendo a cirurgia radical a principal responsável pelo desenvolvimento das alteraçôes físicas (DEVOOGDT et al., 2011a). Em contrapartida, um estudo recente com mulheres mastectomizadas descreveu o desempenho moderado após a cirurgia radical da mama em tais atividades, o que refletiu em uma satisfação regular ou moderadamente boa na sua realização, podendo estas, ainda, influenciar na resposta emocional (BRITO; FONSÊCA, 2014). As cirurgias para tratamento do câncer de mama geralmente alteram a funcionalidade do manguito rotador ao reduzir sua $\mathrm{ADM}$, podendo esta redução ser considerada um preditor de incapacidade do movimento dos MMSS (EBAUGH; SPINELLI; 
SCHMITZ, 2011). Estes resultados corroboram com os achados do presente estudo, que, embora náo tenha avaliado diretamente essa atividade, verificou o comprometimento das atividades laborais e de vida diária devido aos sintomas presentes neste segmento corporal.

No estudo de Smoot et al. (2010), os autores encontraram que as disfunçóes de MMSS são identificadas em mulheres após o tratamento do câncer de mama e constataram que, quanto maior o grau de linfedema, maiores serão as limitaçóes e as repercussóes nas AVD's. Outras complicaçôes encontradas nas mulheres sobreviventes do câncer de mama foram a estrutura da cicatriz, o quadro álgico e a postura protetiva de ombro, com a consequente atrofia dos músculos que compõem a cintura escapular. Geralmente, estas alteraçôes podem ser visíveis em seis meses após o tratamento cirúrgico, o que indica a necessidade de monitoramentos e açôes preventivas, a fim de minimizar a evolução do possível quadro de incapacidade (EBAUGH; SPINELLI; SCHMITZ, 2011).

Essas disfunções previamente descritas dificultam a manutenção da atividade profissional, podendo ter um efeito negativo tanto na qualidade de vida, quanto nos âmbitos econômicos e sociais (BRITO; FONSÊCA, 2014). Deste modo, percebe-se a necessidade crescente de uma ação interdisciplinar, dado que o membro superior e, particularmente, a mão sejam os responsáveis pela independência físico-funcional dos MMSS (DEVOOGDT et al., 2011b; CUESTA-VARGAS; BUCHAN; ARROYO-MORALES, 2014; TESTA et al., 2014).

Em equivalência, particularmente a terapia ocupacional poderá propiciar a elas melhor desempenho e o retorno o mais precoce possível às atividades laborais, de lazer e educativas (HWANG et al., 2015).

\subsection{Limitações do estudo}

A ausência dos dados estatisticamente significativos deve-se ao número reduzido da amostra, explicado pelas perdas relacionadas ao óbito, à mudança do endereço residencial e à não aceitação em fazer parte do estudo. Assim, os resultados encontrados devem ser interpretados com cautela, evitando generalizaçôes.

\subsection{Sugestão para estudos futuros}

Sugere-se que sejam feitos estudos de seguimento, a fim de caracterizar melhor essa população e suas limitaçôes físicas. Tal reflexão permitirá encontrar respostas para um melhor ajuste e/ou manejo nos diferentes tipos ocupacionais (trabalhadoras manuais e não manuais). Em complemento, poder-se-á oferecer ferramentas aos empregadores no processo de adaptação às funções laborais, tendo-se, como base, as condiçóes de saúde, de conforto e de segurança, sem que haja prejuízo na qualidade do processo produtivo.

\section{Conclusão}

Este estudo possibilitou verificar distintos comportamentos após o tratamento, quais sejam: parte dessas mulheres se afastou de suas atividades ocupacionais, foi encaminhada para a aposentadoria ou, ainda, teve de abandonar a atividade profissional, após o tempo de licença saúde. Em relação ao tipo de cirurgia, a maior parte das mulheres realizou algum tipo de cirurgia radical da mama e, quase sua totalidade, passou por linfadenectomia. As queixas físicas mais frequentes foram dor, linfedema, seroma, alteração da ADM, redução da sensibilidade e problemas cicatriciais. Estas complicaçôes, somadas às demandas ocupacionais, contribuíram para o desenvolvimento das modificações de comportamento laboral por elas relatadas. Acredita-se que um acompanhamento multidisciplinar pós-operatório precoce seja essencial na amenização dos sintomas físicos e, consequentemente, auxilie no retorno ao trabalho.

\section{Referências}

BARNES, A. J.; ROBERT, N.; BRADLEY, C. J. Job attributes, job satisfaction and the return to health after breast cancer diagnosis and treatment. Psycho- Oncology, Oxford, v. 23, n. 2, p. 158-164, 2014.

BLINDER, V. S. et al. Return to work in low-income Latina and non-Latina white breast cancer survivors: a 3-year longitudinal study. Cancer, New York, v. 118, n. 6, p. 1664-1674, 2012.

BRITO, J. S.; FONSÊCA, J. Q. M. Desempenho ocupacional de mulheres submetidas à mastectomia/Occupational performance of women subjected to mastectomy. Cadernos de Terapia Ocupacional da UFSCar, Sáo Carlos, v. 22, n. 3, p. 473-485, 2014.

CARLSEN, K. et al. Unemployment among breast cancer survivors. Scandinavian Journal of Public Health, Estocolmo, v. 42, n. 1, p. 319-328, 2014.

CHEN, D. et al. Conservative surgery plus axillary radiotherapy vs. modified radical mastectomy in patients with stage I breast cancer. Clinical Breast Cancer, Dallas, v. 14, n. 1, p. 10-13, 2014.

COOPER, A. F. et al. Distinct work-related, clinical and psychological factors predict return to work following treatment in four different cancer types. Psycho- Oncology, Oxford, v. 22, n. 3, p. 659-667, 2012. 
COWHER, M. S. et al. Conservative axillary surgery in breast cancer patients undergoing mastectomy: long-term results. Journal of the American College of Surgeons, Chicago, v. 218, n. 4, p. 819-824, 2014.

CUESTA-VARGAS, A. I.; BUCHAN, J.; ARROYOMORALES, M. A multimodal physiotherapy programme plus deep water running for improving cancer-related fatigue and quality of life in breast cancer survivors. European Journal of Cancer Care, Oxford, v. 23, n. 1, p. 15-21, 2014.

DEVOOGDT, N. et al. Short-and long-term recovery of upper limb function after axillary lymph node dissection. European Journal of Cancer Care, London, v. 20, n. 1, p. 77-86, 2011 a.

DEVOOGDT, N. et al. Effect of manual lymph drainage in addition to guidelines and exercise therapy on arm lymphoedema related to breast cancer: randomised controlled trial. BMJ, London, v. 343, p. d5326, 2011 b.

DORLAND, H. F. et al. Factors influencing work functioning after cancer diagnosis: a focus group study with cancer survivors and occupational health professionals. Supportive Care in Cancer, Berlim, v. 24, n. 1, p. 261266, 2016.

EBAUGH, D.; SPINELLI, B.; SCHMITZ, K. H. Shoulder impairments and their association with symptomatic rotator cuff disease in breast cancer survivors. Medical Hypotheses, London, v. 77, n. 4, p. 481-487, 2011.

EDWARDS, R. R. et al. Alteration in pain modulation in women with persistent pain after lumpectomy: influence of catastrophizing. Journal of Pain and Symptom Management, New York, v. 46, n. 1, p. 30-42, 2013.

FANTONI, S. Q. et al. Factors related to return to work by women with breast cancer in northern France. Journal of Occupational Rehabilitation, Dordrecht, v. 20, n. 1, p. 49-58, 2010.

HWANG, E. J. et al. Functional deficits and quality of life among cancer survivors: implications for Occupational Therapy in cancer survivorship care. American Journal of Occupational Therapy, Boston, v. 69, n. 6, p. $1-9,2015$.

INSTITUTO NACIONAL DE CÂNCER - INCA. Estimativa 2014: incidência de câncer no Brasil. Rio de Janeiro: INCA, 2014. Disponível em: <http://www.inca. gov.br/rbc/n_60/v01/pdf/11-resenha-estimativa-2014-incidencia-de-cancer-no-brasil.pdf>. Acesso em: 1 maio 2015.

INSTITUTO NACIONAL DE CÂNCER - INCA. Estimativa 2016: incidência de câncer no Brasil. Rio de Janeiro: INCA, 2016. Disponível em: <http://www.inca. gov.br/wcm/dncc/2015/estimativa-2016.asp >. Acesso em: 11 abr. 2017.
INTERNATIONAL AGENCY FOR RESEARCH ON CANCER - IARC. Globocan 2012: estimated cancer incidence, mortality and prevalence worldwide in 2012. Lyon: IARC, 2012. Disponível em: <http://globocan.iarc. fr/Pages/fact_sheets_cancer.aspx>. Acesso em: 1 maio 2015.

ISLAM, T. et al. Factors associated with return to work of breast cancer survivors: a systematic review. $B M C P u$ blic Health, Londres, v. 14, p. 2-13, 2014. Suplemento 3.

LILLIEHORN, S. et al. Meaning of work and the returning process after breast cancer: a longitudinal study of 56 women. Scandinavian Journal of Caring Sciences, Estocolmo, v. 27, n. 2, p. 267-274, 2013.

LINDBOHM, M. L. et al. Early retirement and nonemployment after breast cancer. Psycho- Oncology, Oxford, v. 23, n. 6, p. 634-641, 2014.

MONLEON, S. et al. Lymphedema predictor factors after breast cancer surgery: a survival analysis. Lymphatic Research and Biology, Larchmont, v. 13, n. 4, p. 268-274, 2015.

PYFER, B. et al. Early postoperative outcomes in breast conservation surgery versus simple mastectomy with implant reconstruction: a NSQIP analysis of 11, 645 patients. Annals of Surgical Oncology, New York, v. 23, n. 1 , p. $1-7,2015$.

RHIE, J.; JEONG, I.; WON, J. U. K. Return-to-work according to impairment type among occupationally injured workers in Korea. Journal of Korean Medical Science, Seul, v. 28, n. 11, p. 1581-1586, 2013.

ROELEN, C. A. M. et al. Return to work after cancer diagnosed in 2002, 2005 and 2008. Journal of Occupational Rehabilitation, Dordrecht, v. 21, n. 3, p. 335-341, 2011.

SALONEN, P. et al. Changes in quality of life in patients with breast cancer. Journal of Clinical Nursing, Oxford, v. 20, n. 1-2, p. 255-266, 2011.

SMOOT, B. et al. Upper extremity impairments in women with or without lymphedema following breast cancer treatment. Journal of Cancer Survivorship, New York, v. 4, n. 2, p. 167-178, 2010.

TESTA, A. et al. Strengths of early physical rehabilitation programs in surgical breast cancer patients: results of a randomized control study. European Journal of Physical and Rehabilitation Medicine, Torino, v. 50, n. 3, p. 275-284, 2014.

TIMPERI, A. W. et al. Employment status and quality of life in recently diagnosed breast cancer survivors. Psycho-Oncology, Oxford, v. 22, n. 6, p. 1411-1420, 2013.

TOGAWA, K. et al. Risk factors for self-reported arm lymphedema among female breast cancer survivors: a prospective cohort study. Breast Cancer Research, London, v. 16, n. 4, p. 414-429, 2014. 
TSAI, R. J. et al. The risk of developing arm lymphedema among breast cancer survivors: a meta-analysis of treatment factors. Annals of Surgical Oncology, New York, v. 16, n. 7, p. 1959-1972, 2009.
VERONESI, U. et al. Twenty-year follow-up of a randomized study comparing breast-conserving surgery with radical mastectomy for early breast cancer. New England Journal of Medicine, Melbourn, v. 347, n. 16, p. 1227-1232, 2002.

\section{Contribuição dos Autores}

Mirella Dias foi responsável pela coleta e análise de dados, discussão dos resultados, escrita do texto. Kamilla Zomkowski foi responsável pela escrita do texto, preparo para envio à publicação. Fernanda Alessandra Silva Michels foi responsável pela elaboração do projeto, análise de dados. Fabiana Flores Sperandio foi responsável pela análise de dados, discussão dos resultados, revisão final do artigo. Todos os autores aprovaram a versão final do texto. 